\title{
First transfemoral percutaneous edge-to-edge repair of the tricuspid valve using the MitraClip system
}

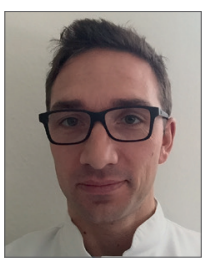

Tobias Wengenmayer ${ }^{1 *}$, MD; Manfred Zehender ${ }^{1}, \mathrm{MD}$; Wolfgang Bothe ${ }^{2}, \mathrm{MD}$; Christoph Bode ${ }^{1}, \mathrm{MD}$; Sebastian Grundmann ${ }^{1}$, MD

1. Department of Cardiology and Angiology I, Heart Center Freiburg University, Freiburg, Germany; 2. Department of Cardiovascular Surgery, Heart Center Freiburg University, Freiburg, Germany

This paper also includes supplementary data published online at: http://www.pcronline.com/eurointervention/97th_issue/296

\section{KEYWORDS}

- MitraClip

- percutaneous

tricuspid valve

repair

- transfemoral

access

- tricuspid

regurgitation

\section{Abstract}

Aims: While severe tricuspid regurgitation contributes significantly to morbidity and a poor prognosis in heart failure patients, isolated surgical repair of the tricuspid valve is associated with a high mortality, especially in patients with prior surgery. Percutaneous tricuspid valve repair could contribute to the solution of this dilemma. A recently published report demonstrated the feasibility of tricuspid edge-to-edge repair with the MitraClip ${ }^{\circledR}$ system (Abbott Vascular, Santa Clara, CA, USA) using a transjugular route. In a highly symptomatic patient with severe functional tricuspid regurgitation unsuitable for surgery, we aimed to reduce tricuspid regurgitation using a modified deployment technique for the MitraClip system using a femoral access.

Methods and results: A 78-year-old male patient with dilated cardiomyopathy was admitted for his fifth episode of acutely decompensated, predominantly right-sided, heart failure in ten months. Echocardiography showed a moderately to severely reduced left ventricular function and severe functional tricuspid regurgitation. Two clips were deployed in the anterior-septal and posterior-septal commissure of the tricuspid valve, resulting in a significant reduction of the effective regurgitant orifice area. The midterm clinical state of the patient improved.

Conclusions: In our patient with severe functional tricuspid regurgitation and suitable anatomy, transfemoral percutaneous tricuspid valve repair was technically feasible and safe by using a modified deployment technique of a widely used repair system developed for the mitral valve. The procedure can be performed without technical modification of the device via a transfemoral route and under echocardiographic guidance. However, particular anatomic and technical aspects can compromise an initial procedural success and demand specific considerations in the future. 


\section{Introduction}

The prevalence of tricuspid regurgitation (TR) in patients with heart failure due to ischaemic heart disease or mitral valve disease is high: one third of patients undergoing mitral valve surgery suffer from significant concomitant regurgitation of the tricuspid valve'. Moreover, one-year survival is significantly lower in patients with severe TR compared to patients without TR $(65 \% \text { vs. } 90 \%)^{2}$. Increasing evidence for a long-term benefit has resulted in a more aggressive approach in many centres performing tricuspid valve repair at the time of mitral valve surgery. However, recurrence rates of significant TR range between $10 \%$ and $45 \%$, and secondary TR repair late after left-sided cardiac surgery carries a high burden of perioperative mortality (around $10 \%$ ). This results in a high number of undertreated patients in great need of alternative therapeutic strategies, including interventional approaches, which could reduce the procedural risk associated with extracorporeal circulation and surgical trauma. Very recently, Hammerstingl and co-workers demonstrated that a transcatheter edge-to-edge repair of the tricuspid valve is feasible from the transjugular route ${ }^{3}$. In the current report, we describe our first case experience with the attempt to reduce tricuspid valve regurgitation with the MitraClip system (Abbott Vascular, Santa Clara, CA, USA) via a transfemoral access.

\section{Case description}

A 78-year-old patient with recurrent right heart decompensations was admitted to our department with advanced New York Heart Association Class III symptoms. It was his fifth episode of acutely decompensated heart failure in ten months. He presented with cardiorenal syndrome type I, ascites and third-degree peripheral oedema despite well-established heart failure therapy, including a cardiac resynchronisation therapy device. Echocardiography showed a moderately to severely reduced left ventricular function, a mild to moderate mitral regurgitation and severe functional TR with an effective regurgitant orifice area (EROA) of $1.0 \mathrm{~cm}^{2}$, regurgitation volume $96 \mathrm{ml}$, and flow reversal in the hepatic veins (Figure 1A, Figure 1B). TR was due to annular dilatation and missing coaptation of the leaflets. The right atrium and ventricle were dilated with impaired right ventricular function. His renal function was severely impaired (creatinine $3.4 \mathrm{mg} / \mathrm{dl}$, glomerular filtration rate $18 \mathrm{ml} / \mathrm{min}$ ). A surgical tricuspid valve repair was considered to be high risk (EuroSCORE $45 \%$ ). With the futility of conservative treatment and with the lack of alternative treatment options the patient agreed to an attempt at percutaneous edge-toedge repair of the tricuspid valve.

Prior to intervention, transoesophageal echocardiography (TOE) images were acquired to ensure a sufficient acoustic window for detailed analysis of the tricuspid valve and for guiding the catheter system in the right atrium. The TOE images confirmed a missing coaptation in the septal-posterior and septal-anterior commissure, resulting in a severe TR. The interventional reconstruction was performed under general anaesthesia with fluoroscopic and echocardiographic guidance.
The deflectable MitraClip guide catheter was introduced via the femoral route into the right atrium. The clip could be advanced approximately $20 \mathrm{~mm}$ outside the guide until it reached the tricuspid annular plane. Thus, specific steering mechanisms of the system $(\mathrm{M}+\mathrm{L})$ were not available, as the clip catheter cannot be deflected inside the guide catheter.

Using a transgastric tricuspid valve short-axis view, the clip was positioned perpendicular to the septal-anterior commissure (Figure 1C). A modified four-chamber view was used to image the clip, the septal and anterior leaflets. Views were adjusted according to clip rotation. Using this modified midoesophageal fourchamber view, several grasping attempts were made at varying distances from the tricuspid ring. Placing a clip between the septal and anterior leaflets resulted in an almost complete reduction of the regurgitation in that commissure, creating a double orifice (Figure 1D-Figure 1F). As the regurgitation in the septal-posterior commissure increased, we placed a second clip in the septal-posterior commissure, which reduced TR from severe to mild, creating a triple orifice tricuspid valve (Figure 1G-Figure 1J). Mean gradient over the TV was $1 \mathrm{mmHg}$. The clip manoeuvres were guided by TOE exclusively. The patient was extubated in the cathlab with an uneventful post-interventional recovery.

Three days later, transthoracic echocardiography showed an again increased TR, most likely due to a single leaflet attachment of the second clip. Regurgitation volume was assessed with $64 \mathrm{ml}$ (EROA $0.5 \mathrm{~cm}^{2}$ ) using the PISA method.

The patient was discharged home six days after the intervention. In his follow-up examination 40 days later, our patient reported an improved overall wellbeing. Echocardiography showed a stable result with a TR volume of $46 \mathrm{ml}\left(\right.$ EROA $\left.0.49 \mathrm{~cm}^{2}\right)$. His creatinine had reduced from 3.4 to $1.75 \mathrm{mg} / \mathrm{dl}$ and the six-minute walk test demonstrated an increased exercise tolerance as the walking distance improved from 40 to 170 metres.

\section{Discussion}

In our case, percutaneous edge-to-edge repair of the tricuspid valve could be performed with good procedural success. Although there have been rumours about attempts to use the MitraClip device for tricuspid repair for quite some time it was only very recently that Hammerstingl et al reported a first three-case series ${ }^{3}$. In contrast to their transjugular approach we attempted to use a transfemoral access. This might offer the opportunity to address mitral and tricuspid valves in one session in the near future. Therefore, we decided to share our first-in-man experience with the interventional community.

Obviously our report on a single patient cannot provide any evaluation of the efficacy of the technique, but our case gives valuable insights into the steering options, imaging views and technical aspects of the use of MitraClip on "the right side of the heart".

Our aim was to resemble an established surgical repair method, namely the edge-to-edge "clover" technique, in which the middle parts of the free leaflet margins are sutured together, creating a double or triple orifice valve $e^{4}$. Most often, this technique is combined with an annuloplasty, but it can also be performed in isolation ${ }^{5}$. Our 


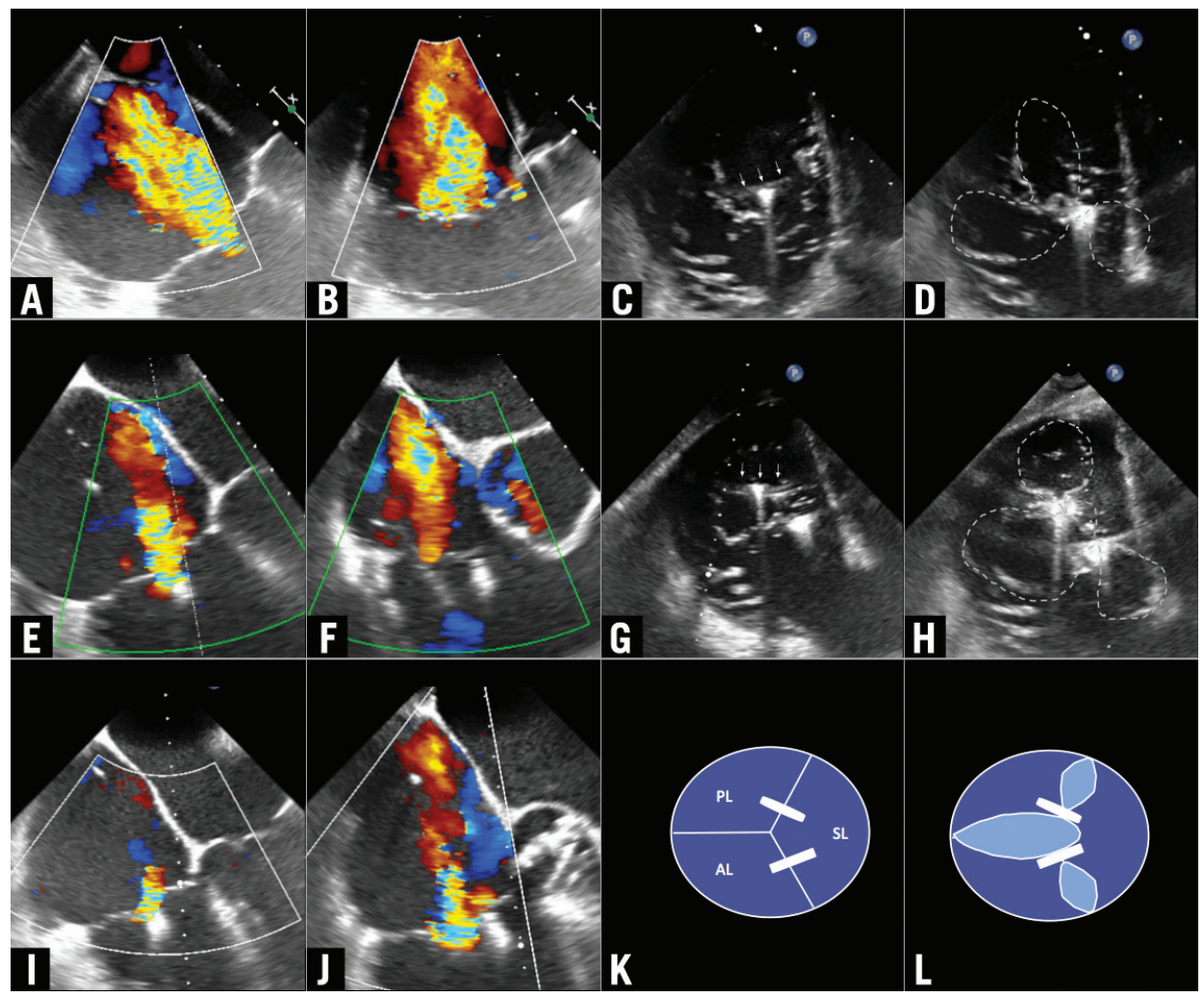

Figure 1. Four-chamber and midoesophageal inflow-outflow views pre, post first clip and second clip ( $A \& B, C \& D, E \& F) ;$ schematic view of valve and clips $(G \& H)$; clip orientation orthogonal to anterior-septal commissure (I); transgastric short-axis view post first clip (J); clip orientation orthogonal to posterior-septal commissure (K); transgastric short-axis view post second clip $(L)$.

two clips resulted in a similar triple orifice valve, obviously without addressing the tricuspid annulus itself (Figure 1K, Figure 1L).

Although the steerable guide catheter of the MitraClip has limited options for angulation and navigation, steering of the device towards the tricuspid valve and below the tricuspid annulus worked surprisingly well via a femoral access. Moving echocardiographic images of the procedure, in chronological order, can be found in the Supplementary data section (Moving images 1-16).

In contrast to device navigation for treatment of the mitral valve, the medial-lateral dials have to be neglected. To position the clip above the individual commissures, imaging in a transgastric shortaxis view allows the best view of the clip position relative to the regurgitant orifice. Advancing or retracting the whole system positions the clip towards the posterior or anterior leaflet, respectively. Counter-clockwise rotation of the delivery system moves the clip towards the septum, clockwise towards either the posterior or the anterior leaflet, depending on how far the system is advanced or retracted. Turning the \pm knob towards $(+)$ results in a posteriorseptal movement of the clip, whereas turning it towards $(-)$ results in an anterior-septal movement.

As in treatment of the mitral valve, imaging is critical for the procedure. In our single case experience, TOE yielded satisfactory imaging quality for navigation of the device by switching mainly between a transgastric short-axis and either a modified midoesophageal four-chamber, a midoesophageal inflow-outflow, or a transgastric long-axis view. However, direct visualisation of the grippers and leaflet attachment during device deployment is inferior compared to the mitral valve. This represents a possible explanation for the single leaflet attachment of one clip which we detected three days after the procedure, as we could not directly visualise the length of leaflet attachment during device deployment. In future, intracardiac ultrasound could possibly offer adjuvant imaging techniques to overcome these obstacles.

\section{Conclusion}

Our case demonstrates that reduction of severe TR using the MitraClip device can be technically feasible with sufficient steering abilities using the femoral route. Future case studies with the currently available systems as well as comparisons to other interventional techniques will have to show whether percutaneous tricuspid valve repair could evolve as a possible treatment option for these patients.

\section{Impact on daily practice}

Percutaneous tricuspid valve repair with the MitraClip device is feasible and effective using a transfemoral access and offers a treatment option for symptomatic high-risk patients. Percutaneous tricuspid valve repair is at an early stage and various upcoming interventional techniques have to be evaluated in the near future. 


\section{Conflict of interest statement}

The authors have no conflicts of interest to declare.

\section{References}

1. Cohen SR, Sell JE, McIntosh CL, Clark RE. Tricuspid regurgitation in patients with acquired, chronic, pure mitral regurgitation. II. Nonoperative management, tricuspid valve annuloplasty, and tricuspid valve replacement. J Thorac Cardiovasc Surg. 1987;94:488-97.

2. Nath J, Foster E, Heidenreich PA. Impact of tricuspid regurgitation on long-term survival. J Am Coll Cardiol. 2004; 43:405-9.

3. Hammerstingl C, Schueler R, Malasa M, Werner N, Nickenig G. Transcatheter treatment of severe tricuspid regurgitation with the MitraClip system. Eur Heart J. 2016 Jan 7. [Epub ahead of print].

4. Lapenna E, De Bonis M, Maisano F, Crescenzi G, La Canna G, Torracca L, Grimaldi A, Alfieri O. 'Four-leaflet clover repair' of severe tricuspid valve regurgitation due to complex lesions. J Cardiovasc Med (Hagerstown). 2008;9:847-9.

5. Lapenna E, De Bonis M, Verzini A, La Canna G, Ferrara D, Calabrese MC, Taramasso M, Alfieri O. The clover technique for the treatment of complex tricuspid valve insufficiency: midterm clinical and echocardiographic results in 66 patients. Eur $J$ Cardiothorac Surg. 2010;37:1297-303.

\section{Supplementary data}

Moving image 1. Bicaval view with guide catheter pointing in direction of tricuspid valve.

Moving image 2. Bicaval view with $1^{\text {st }}$ clip advanced.

Moving image 3. Four-chamber view, TR pre-intervention.

Moving image 4. Inflow-outflow view, TR pre-intervention.

Moving image 5. Transgastric short axis, TR pre-intervention.

Moving image 6. Long and short axis (x-plane), $1^{\text {st }}$ clip orientation.

Moving image 7. Four-chamber view, $1^{\text {st }}$ clip opened, before grasping.

Moving image 8. Transgastric short axis, $1^{\text {st }}$ clip orientation not orthogonal to septal-anterior commissure.

Moving image 9. Transgastric short axis, $1^{\text {st }}$ clip orientation orthogonal to septal-anterior commissure.

Moving image 10. Transgastric short axis, post $1^{\text {st }}$ clip.

Moving image 11. Four-chamber view, TR post $1^{\text {st }}$ clip.

Moving image 12. Transgastric short axis, $2^{\text {nd }}$ clip orientation orthogonal to septal-posterior commissure.

Moving image 13. Four-chamber view, TR post $2^{\text {nd }}$ clip.

Moving image 14. Inflow-outflow view, TR post $2^{\text {nd }}$ clip.

Moving image 15. Transgastric short axis, post $2^{\text {nd }}$ clip.

Moving image 16 . Four-chamber view, post $2^{\text {nd }}$ clip.

The supplementary data are published online at:

http://www.pcronline.com/

eurointervention/97th issue/296 